09

\title{
Расчет терагерцевого лазера в режиме блоховских осцилляций
}

\author{
(С) К.Ю. Голеницкий, А.М. Монахов, В.И. Санкин
}

Физико-технический институт им. А.Ф. Иофре РАН, Санкт-Петербург, Россия

E-mail: golenitski.k@mail.ioffe.ru

Поступило в Редакцию 6 августа 2018 г.

Рассмотрены возможные конструкции терагерцевого лазера, использующего эффект блоховских осцилляций в естественных сверхрешетках $\mathrm{SiC}$. Предлагается использовать сам кристалл $\mathrm{SiC}$, на котором выращен активный слой, в качестве резонатора. Рассчитаны размеры резонаторов для полоскового лазера и лазера, работающего на модах шепчущей галереи. Показаны преимущества резонатора с модами шепчущей галереи.

DOI: 10.21883/PJTF.2018.24.47032.17493

Создание приборов терагерцевого $(\mathrm{THz})$ диапазона - крайне актуальная задача, которой посвящено огромное число публикаций. Они находят применение во всех областях науки и техники. Запрос по этой теме выдает более ста тысяч ссылок, привести которые нет никакой возможности. Для того чтобы понять охват различных областей, достаточно взглянуть на современный обзор научно-технологической карты о THz-диапазоне [1].

Одним из перспективных путей создания таких приборов является использование режима блоховских осцилляций для генерации THz-излучения. Эта идея восходит к работам Блоха [2] и Зинера [3] 20-30-х годов XX века и состоит в том, что если энергия электрона $\varepsilon(k)$ является периодической функцией квазиимпульса $k$, то в постоянном достаточно сильном электрическом поле $E$ координата электрона будет осциллировать в реальном пространстве с частотой

$$
\omega_{B O}=\frac{e E a}{\hbar},
$$

где $a-$ постоянная решетки кристалла. Такой колеблющийся электрон должен будет излучать электромагнитные волны с частотой $\omega_{B O}$. 
Дальнейшие теоретические исследования [4] показали, что наличие блоховских осцилляций приводит к оптическому усилению на частотах ниже $\omega_{B O}$ с максимумом на частоте чуть меньше $\omega_{B O}$.

В течение 90 лет, прошедших с момента предсказания явления генерации излучения в сверхрешетках, были предприняты попытки экспериментально обнаружить такое излучение [4-6]. Возникшие экспериментальные сложности связаны с тем, что время рассеяния носителей должно быть больше, чем период блоховских осцилляций. Кроме того, отрицательное сопротивление на постоянном токе приводит к возникновению доменной структуры электрического поля. Все эти эффекты особенно выражены в неидеальных сверхрешетках, выращенных на основе традиционных полупроводниковых структур.

Естественные сверхрешетки, возникающие вследствие не до конца изученных причин в гексагональных модификациях карбида кремния, являются весьма перспективным объектом для создания излучающих приборов, использующих эффект осцилляций Блоха. Качество таких естественных сверхрешеток существенно превосходит качество искусственно выращенных структур. В недавних работах [7] было получено THz-излучение из таких материалов при прямой накачке постоянным током. Данные, приведенные в [7], позволяют с большой уверенностью предполагать, что это излучение вызвано блоховскими осцилляциями носителей в естественной сверхрешетке $\mathrm{SiC}$. Характерный спектр спонтанного излучения в режиме генерации блоховских осцилляций в таких сверхрешетках при пороговом напряжении приведен на рис. 1. В этом случае есть основания надеяться на создание полупроводникового лазеpa, поскольку известно, что в сверхрешетках имеет место оптическое усиление на частоте, близкой к частоте блоховских осцилляций.

Существенным элементом лазера является оптический резонатор. В случае обычных полупроводниковых лазеров оптического и среднего ИК-диапазона размеры резонатора, по крайней мере вдоль одного измерения, много больше длины волны, что позволяет не особенно заботиться о геометрии резонатора, поскольку на кривой усиления укладывается много резонаторных мод, и „лазер сам выберет“, на какой из них работать. Это не так в THz-диапазоне. В этом случае длина волны остается сравнимой с размером резонатора вплоть до размеров порядка $0.5 \mathrm{~mm}$. Кроме того, имеющаяся в настоящее время технология выращивания структур $\mathrm{SiC}$ не позволяет создавать в них ограничивающие слои с разным показателем преломления. Более того,

Письма в ЖТФ, 2018, том 44, вып. 24 


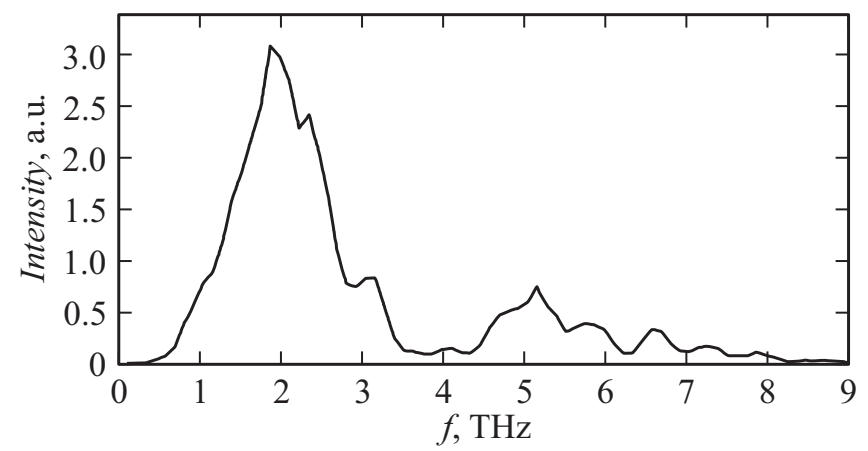

Рис. 1. Экспериментально измеренный спектр электролюминесценции при пороговом напряжении генерации блоховских осцилляций в $6 \mathrm{H}-\mathrm{SiC}$.

к активной области необходимо приложить значительное напряжение. Все это делает конструкцию резонатора такого прибора существенно отличающейся от традиционных. Настоящая работа посвящена рассмотрению возможных конструкций резонаторов для лазеров на основе $\mathrm{SiC}$.

B THz-диапазоне в качестве резонатора предполагается использовать сам кристалл. В простейшем случае [7] излучение в таком кристалле возникает в области, где напряженность электрического поля превышает критическую, необходимую для возникновения блоховских осцилляций. Эта область является слаболегированной и не может иметь размеры более нескольких микрометров $(3-5 \mu \mathrm{m}$ в работе [7]). Кроме того, по технологическим причинам она располагается на расстоянии порядка $10 \mu \mathrm{m}$ от поверхности образца. Резонатор в свою очередь должен иметь размеры порядка сотен микрометров. Поэтому для расчета собственных состояний такого резонатора необходимо проводить полноволновый расчет.

Как известно, простейшей конструкцией полупроводникового лазера является полосковый лазер. В нашем случае нельзя использовать основную моду резонатора, так как активная зона не может быть помещена в середину резонатора. Среди всех мод с частотами в диапазоне усиления подходящими будут только те, у которых максимум распределения электрического поля, параллельного плоскости активной области, расположен в самой активной области. Это условие сильно сокращает число мод, на которых возможна лазерная генерация.

Письма в ЖТФ, 2018, том 44, вып. 24 
В общем случае подбор размеров резонатора сводится к задаче поиска собственных состояний электромагнитного поля и их последующего отбора по перечисленным выше критериям.

Для качественного описания модовой карты полоскового резонатора рассмотрим диэлектрический прямоугольный резонатор с идеальными металлическими стенками с размерами $L_{x}, L_{y}, L_{z}$. Собственные частоты такого резонатора равны

$$
\omega_{m n l}=\frac{c}{\sqrt{\varepsilon}} \sqrt{\frac{\pi^{2} m^{2}}{L_{x}^{2}}+\frac{\pi^{2} n^{2}}{L_{y}^{2}}+\frac{\pi^{2} l^{2}}{L_{z}^{2}}} .
$$

Если $L_{x}>L_{z}>L_{y}$, то частота основной моды $\omega_{101}=\frac{c}{\sqrt{\varepsilon}} \sqrt{\frac{\pi^{2}}{L_{x}^{2}}+\frac{\pi^{2}}{L_{z}^{2}}}$. Диэлектрическая проницаемость $\mathrm{SiC} \varepsilon \cong 10$ в длинноволновом диапазоне. Частота основной моды для резонатора размером $250 \times 100 \times 160 \mu \mathrm{m}$ равна $0.35 \mathrm{THz}$, а пик усиления рассматриваемого образца [7] приходится на область частот $1.2-1.9 \mathrm{THz}$ (рис. 1). Эти частоты сравнимы, что указывает на необходимость точного подбора геометрии кристалла, чтобы удовлетворить приведенным выше условиям. В реальных условиях создать резонатор с металлическими стенками, близкими к идеальным, для THz-диапазона невозможно. Это объясняется тем, что толщина скин-слоя в THz-диапазоне сравнима с длиной волны и, следовательно, с размерами резонатора. Однако, как показано далее, эта простая модель неплохо описывает моды в реальном диэлектрическом резонаторе.

Для реального резонатора его собственные состояния искались численными методами путем решения уравнений Максвелла на трехмерной сетке с помощью пакета COMSOL. На рис. 2 изображены сечения распределения $\sqrt{\left|E_{x}\right|^{2}+\left|E_{y}\right|^{2}}$ (модуля электрического поля, параллельного плоскости активного слоя) в прямоугольном резонаторе размером $370 \times 90 \times 186 \mu \mathrm{m}$ на основе $\mathrm{SiC}$ для моды, подходящей под требуемые условия. Судя по распределению поля, можно сделать вывод, что она отвечает числам $m, n, l=7,1,5$ для резонатора с идеальными металлическими стенками. Соответствующая частота $f_{715}=1.64 \mathrm{THz}$ на $10 \%$ отличается от частоты похожей моды $f=(1.47+0.004 i) \mathrm{THz}$ в открытом резонаторе без металлических стенок, что демонстрирует неплохое согласие простейшей модели с численным решением. Активная область в выращенном кристалле должна находиться в боковом максимуме распределения поля по оси $Z$ на глубине $8 \mu \mathrm{m}$. Излучение из

Письма в ЖТФ, 2018, том 44, вып. 24 

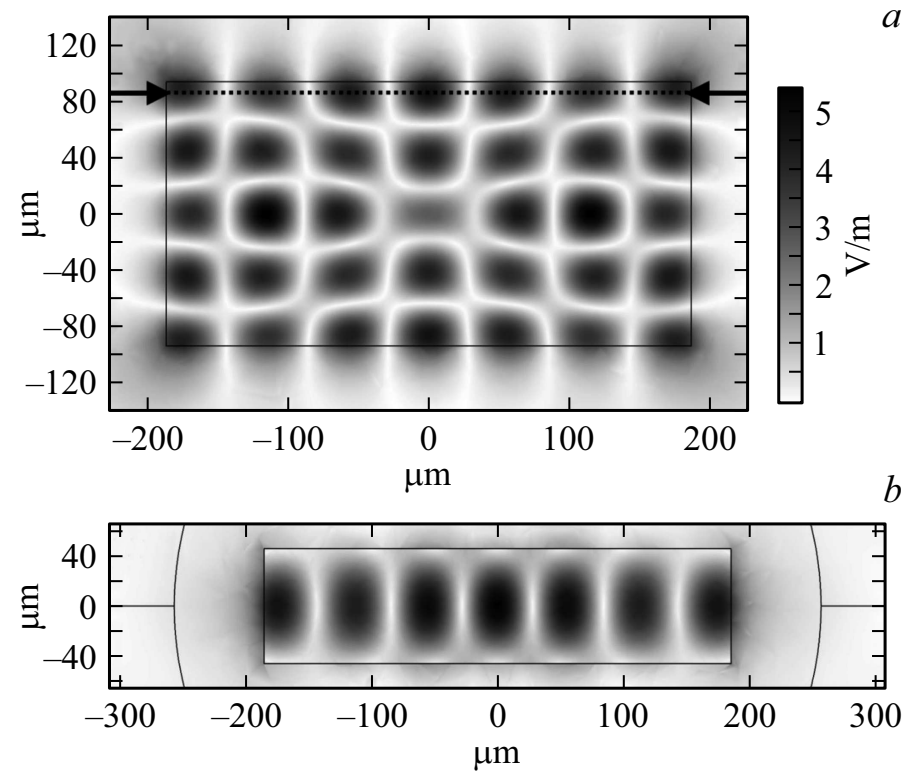

Рис. 2. Сечения распределения $\sqrt{\left|E_{x}\right|^{2}+\left|E_{y}\right|^{2}}-$ модуля электрического поля, параллельного плоскости активного слоя, в прямоугольном резонаторе для моды с частотой $f=(1.47+0.004 i) \mathrm{THz}$. Стрелкой и пунктирной линией отмечено положение активного слоя на глубине $8 \mu \mathrm{m}$. $a-$ сечение плоскостью $y=0, b-$ сечение плоскостью активного слоя.

резонатора для данной моды выводится из торцов $Y Z$. При помещении такого резонатора на подложку возможно существенное утекание моды в нее, что приведет к существенному уменьшению добротности резонатора. Большая добротность резонатора важна в рассматриваемом случае, поскольку усиление прибора невелико, в частности из-за малой толщины активной области. Таким образом, существует необходимость использовать резонатор с наибольшей добротностью.

Добротность открытого резонатора, как известно, определяется двумя факторами: утеканием моды из резонатора и внутренними потерями в резонаторе. Наш расчет показывает, что уже потери за счет утекания (мнимая часть частоты) достаточно велики. Кроме того, существуют и внутренние потери в резонаторе, связанные с поглощением излучения в

Письма в ЖТФ, 2018, том 44, вып. 24 


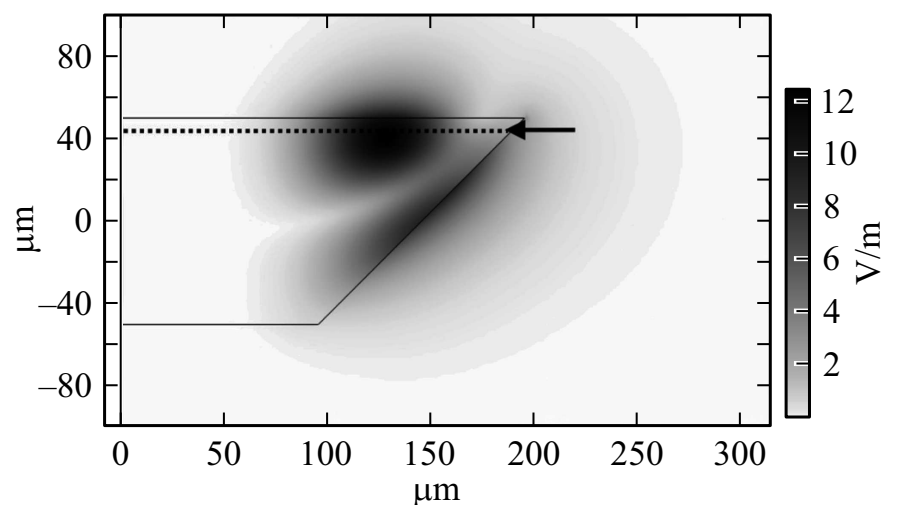

Рис. 3. Распределение $\sqrt{\left|E_{x}\right|^{2}+\left|E_{y}\right|^{2}}$ - модуля поля в плоскости активного слоя для резонатора в форме усеченного конуса для моды с частотой $f=1.49 \mathrm{THz}$ и азимутальным числом $m=10$. Стрелкой и пунктирной линией отмечено положение активного слоя на глубине $9 \mu \mathrm{m}$.

полупроводнике. В полосковом лазере излучение занимает весь объем резонатора и отношение „области усиления“ к „области поглощения“ весьма мало. Поэтому перспектива полоскового лазера на основе $\mathrm{SiC}$, использующего эффект блоховских осцилляций представляется весьма туманной.

Резонаторы с модами шепчущей галереи, обладающие большими величинами добротности, кажутся нам более перспективными. В работе [8] было показано, что в конических резонаторах происходит самоограничение поля вблизи большего основания. Это существенно уменьшает утекание моды в подложку и поэтому позволяет изготовить резонатор сразу на подложке. Кроме того, расчеты показывают, что добротность такого резонатора на порядки превосходит добротность полоскового резонатора, допуская возможность получения лазерной генерации при минимальных коэффициентах усиления. Кроме того, в таком резонаторе можно подобрать моду, которая будет локализована вблизи активной области, что понизит и внутренние потери резонатора. Далее приведены результаты численного расчета такого резонатора для образцов, использовавшихся в [7].

Аналитические формулы для оценки собственных частот из работы [8] не применимы в нашем случае, так как характерные азиму-

Письма в ЖТФ, 2018, том 44, вып. 24 
тальные числа $m<12$ и угол наклона стенки конуса составляет $45^{\circ}$. На рис. 3 представлено рассчитанное численно сечение распределения $\sqrt{\left|E_{x}\right|^{2}+\left|E_{y}\right|^{2}}$ (модуля электрического поля, параллельного плоскости активного слоя) для моды с азимутальным числом $m=10$ и частотой $f=1.49 \mathrm{THz}$, удовлетворяющей требуемым критериям. В пределах точности расчетов $\operatorname{Im} f=0$, что свидетельствует о большей добротности, чем в случае полоскового резонатора. Размеры резонатора: радиус верхнего основания $195 \mu \mathrm{m}$; угол раствора конуса определяется технологическим процессом и равен 90; высота должна быть больше $100 \mu \mathrm{m}$. Максимум поля в резонаторе располагается на глубине $9 \mu \mathrm{m}$, что удобно при росте активной зоны. Частота моды слабо зависит от высоты резонатора; следовательно, влиянием подложки можно пренебречь. Такая геометрия резонатора более предпочтительна по сравнению с полосковым резонатором.

В работе рассмотрены возможные конфигурации резонаторов для терагерцевых лазеров, использующих эффект блоховских осцилляций в $6 \mathrm{H}$-SiC. Допустимые размеры резонаторов ограничены из-за технологических проблем роста кристаллов $\mathrm{SiC}$ и последующей обработки. Классические конфигурации резонаторов в микрополосковых полупроводниковых лазерах не могут быть использованы в этом случае, так как все размеры резонатора сравнимы с длиной волны излучения. Предлагается использовать в качестве резонатора сам кристалл $\mathrm{SiC}$, на котором выращивается активная зона. Рассмотрены две конфигурации резонатора: прямоугольная и в форме усеченного конуса. При точном подборе размеров резонатора в них существуют моды, на которых допускается лазерная генерация. Резонаторы, в которых имеется дополнительное пространственное самоограничение (как в резонаторах в форме усеченного конуса), более предпочтительны. Приведены конкретные размеры резонаторов для структур из работы [7], в которых наблюдалось спонтанное излучение в режиме блоховских осцилляций вблизи $1.5 \mathrm{THz}$.

Авторы выражают благодарность А.Н. Баранову (университет Монпелье-2) за предоставленную возможность воспользоваться пакетом COMSOL.

Работа выполнена при финансовой поддержке РФФИ (грант № 18-02-00208) и Правительства РФ (контракт 14.W03.31.0011 в ФТИ им. А.Ф. Иоффе).

Письма в ЖТФ, 2018, том 44, вып. 24 


\section{Список литературы}

[1] Dhillon S.S., Vitiello M.S., Linfield E.H., Davies A.G., Hoffmann M.C., Booske J., Paoloni C., Gensch M., Weightman P., Williams G.P., Castro-Camus E., Cumming D.R.S., Simoens F., Escorcia-Carranza I., Grant J., Lucyszyn S., Kuwata-Gonokami M., Konishi K., Koch M., Schmuttenmaer C.A., Cocker T.L., Huber R., Markelz A.G., Taylor Z.D., Wallace V.P., Zeitler J.A., Sibik J., Korter T.M., Ellison B., Rea S., Goldsmith P., Cooper K.B., Appleby R., Pardo D., Huggard P.G., Krozer V., Shams H., Fice M., Renaud C., Seeds A., Stöhr A., Naftaly M., Ridler N., Clarke R., Cunningham J.E., Johnston M.B. // J. Phys. D: Appl. Phys. 2017. V. 50. P. 043001. DOI: 10.1088/1361-6463/50/4/043001

[2] Bloch F. // Z. Phys. 1929. V. 52. P. 555-600.

[3] Zener C. // Proc. Roy. Soc. Lond. A. 1934. V. 145. P. 523-529.

[4] Ктиторов С.А, Симин Г.С., Синдаловский В.Я. // ФТТ. 1971. Т. 13. В. 8. C. 2230-2233.

[5] Waschke C., Roskos H.C., Schwedler R., Leo K., Kurz H., Kohler K. // Phys. Rev. Lett. 1993. V. 70. P. 3319-3322.

[6] Kuehn W.,Gaal P., Reimann K., Woerner M., Elsaesser T., Hey R. // Phys. Rev. Lett. 2010. V. 104. P. 146602.

[7] Sankin V.I., Andrianov A.V., Zakharin A.O., Petrov A.G. // Appl. Phys. Lett. 2012. V. 100. P. 111109.

[8] Алексеенко Я.В., Монахов А.М., Рожсанский И.В. // ЖТФ. 2009. Т. 79. В. 11. C. $72-76$. 